\title{
Laserforming of 3D Features
}

\author{
J. R. Duflou ${ }^{a}$, B. Callebaut ${ }^{\mathrm{b}}$ and J.-P. Kruth ${ }^{\mathrm{c}}$ \\ Department of Mechanical Engineering, Katholieke Universiteit Leuven, Belgium \\ aJoost.Duflou@mech.kuleuven.ac.be, `Jean-Pierre.kruth@mech.kuleuven.ac.be, \\ bBart.Callebaut@mech.kuleuven.ac.be
}

Keywords: laser, forming, 3D features

\begin{abstract}
The aim of the research presented in this paper is to investigate the feasibility of generating 3D features in sheet metal blanks using laser forming techniques. Aiming for systematic process planning and a good process control capability, only the temperature gradient mechanism is considered for this purpose.

The research has been performed on a $6 \mathrm{~kW} \mathrm{CO}$ laser $\mathrm{CNC}$ platform. In a first part, bending along curved lines and bending close to edges are investigated. In a second part, these findings are applied for the creation of a louver. A dimensional measurement system is used for analyzing the geometric dimensional characteristics of the formed shapes. It is found that louvers can be made as a local 3D feature in a sheet metal blank.

Based on these initial results, it can be concluded that form features, which are traditionally generated by means of form tools on a punching machine, can to some extend also be obtained by means of industrial lasers, widening the application range of these machine tools.
\end{abstract}

\section{Introduction}

The basic idea of laser forming is to start from a flat metal sheet or an intermediate form and to induce thermal stresses by irradiating the sheet with a laser beam in order to obtain a residual deformation after cooling. Different mechanisms have been identified [1,2], of which the temperature gradient mechanism (TGM), the buckling mechanism (BM) and the upsetting mechanism (UM) are the most relevant. The former two cause a deformation out-of-plane, while the latter deforms in-plane.

Since Namba in the mid-eighties presented the first findings about laser forming [3], 2D forming or bending of metal sheets by laser irradiation has been explored intensively and is quite well under control [e.g., 4-6]. However, the findings of this 2D forming can not easily be extrapolated to $3 \mathrm{D}$ forming, which is much more difficult to control. Although progress has been made in this area in recent years [7-10], more research is needed to achieve a full understanding and to clearly prove the applicability of 3D forming in an industrial context.

In this article, a 3D experimental study is reported aimed at detecting the influence on the bending angle of both the curvature and the distance between scan line and a part edge. This knowledge is then applied for a possible industrial application: the incorporation of a louver in a flat metal sheet. Since the louvers inside this proceeding, are made with a common 3-axis industrial $\mathrm{CO}_{2}$ laser cutting machine, this application raises the flexibility of such machines. It will be of interest to apply the technique to customised louvers.

\section{Experimental Set-Up}

Hardware specifications. For all tests a gantry type laser cutting machine has been used. For the tests to determine the influence of both the curvature and the distance to the cut on the bending angle, the used settings are listed in Table 1. 
Table 1. Overview of process parameters and settings

\begin{tabular}{|l|l|}
\hline Process parameters & Setting \\
\hline Total equivalent laser power $\mathrm{P}_{\mathrm{e}}:$ & \\
Laser power $\mathrm{P}$ & $6 \mathrm{~kW}$ \\
Frequency $\mathrm{f}$ & $1000 \mathrm{~Hz}$ \\
Duty-cycle DC & $60 \%$ \\
\hline Velocity v & $5000 \mathrm{~mm} / \mathrm{min}$ \\
\hline Spot diameter $\mathrm{d}$ & $2.5 \mathrm{~mm}$ \\
\hline Lens & $10 \mathrm{inch}$ \\
\hline
\end{tabular}

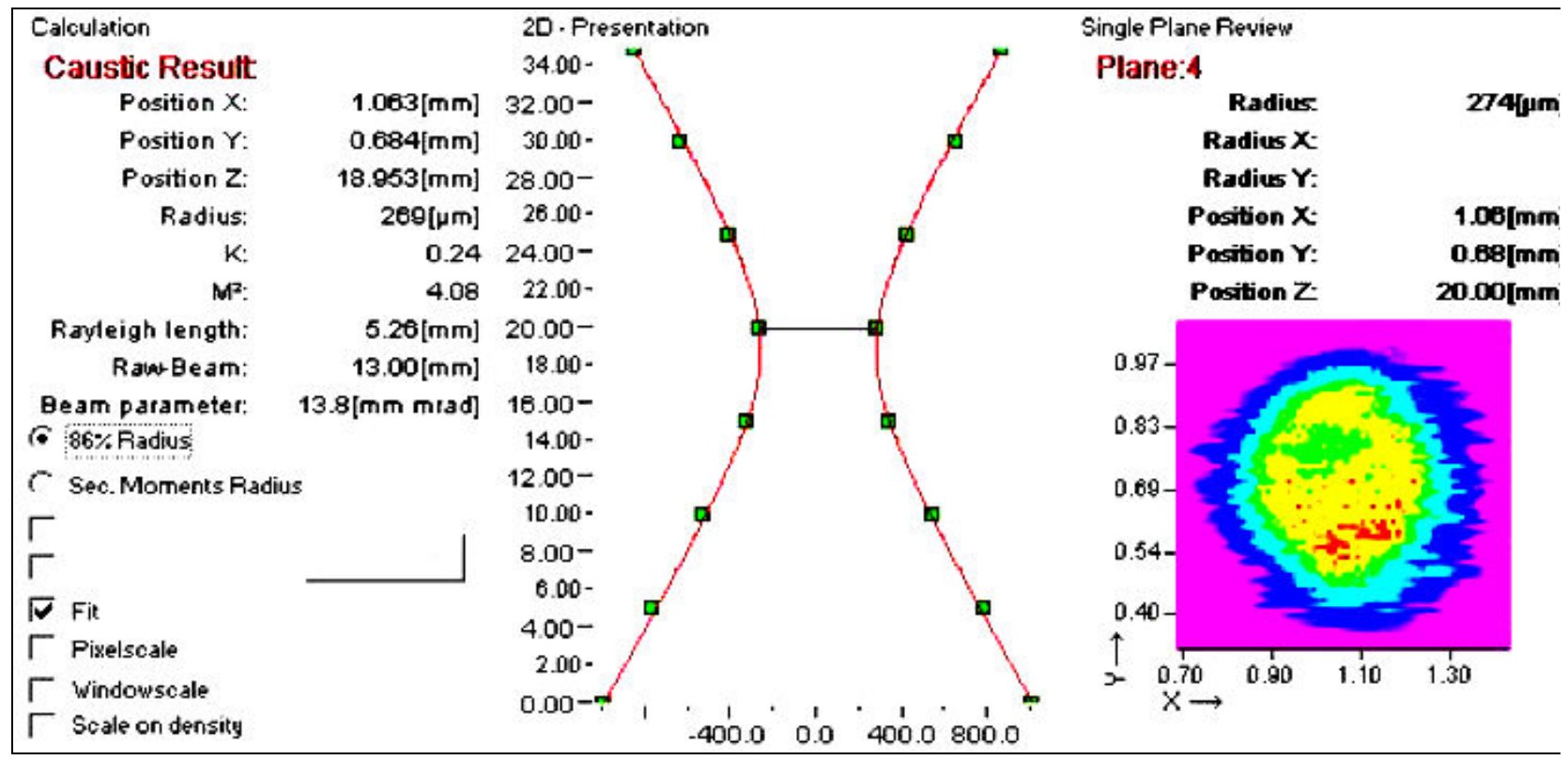

Fig. 1. Beam characteristics as measured with a Primes laser beam analyser

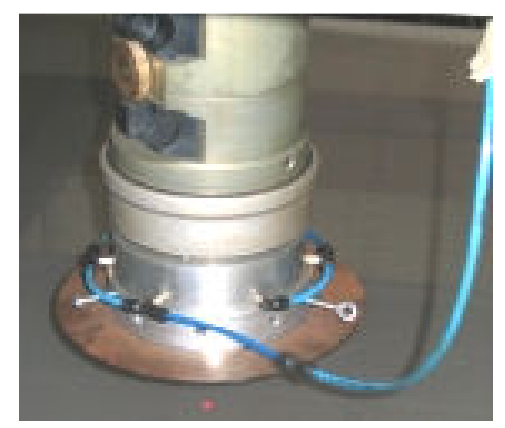

Fig. 2. Laser cutting head with cooling devices

The Fanuc $6 \mathrm{~kW}$ CO2 laser source has beam characteristics as shown in Figure 1

Since during experiments both the metal sheet and the nozzle (by the reflections) are heated, special precautions have to be taken. To cool the nozzle with pressurised air, a specially designed cooling device is mounted on the laser cutting head, and to prevent that light reflections can reach the nozzle, a heatshield is mounted on that cooling device. This heat-shield absorbs or deflects most of the reflected light. Figure 2 shows the laser cutting head with the cooling device and heat shield.

To avoid heat accumulation in the material, a waiting time of

30 seconds is applied between consecutive scans.

Test parts, test procedure and measuring method. The tested material is stainless steel AISI304L of $1.021 \pm 0.008 \mathrm{~mm}$ thickness and its chemical composition is given in Table 2 .

Table 2. Chemical composition of AISI304L (wt.\%)

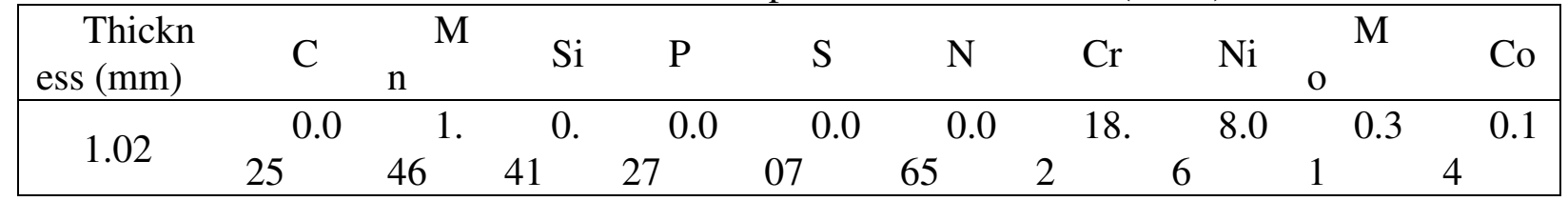


The absorption coefficient for $\mathrm{CO}_{2}$ laser light for this material is somewhere near $10 \%$ [2]. Furthermore, the samples were not specially treated, but scanned as received. The geometry of the test parts is shown in Figure 3. Note that the order of cutting and scanning has been indicated and that bold and dotted lines represent cutting and laser scanning lines respectively. Each test has been repeated at least 3 times. Each scan line is scanned twice. The sheet metal blank is put in a horizontal position by means of 4 adjustable screws, one on each foot of the supporting table, and

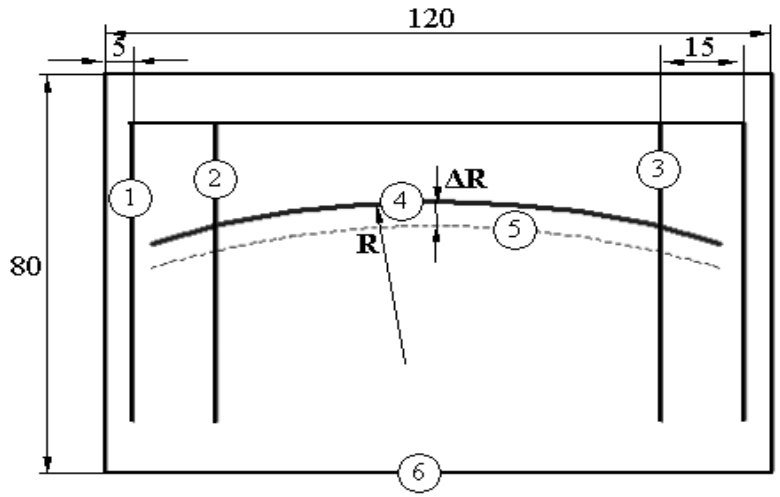

Fig. 3. Test part layout. Bold lines are cut, dotted lines are scan lines problems with changing stand-off distances along the bending lines are thus avoided. Scanning line 5 has a smaller radius than cut 4 . The difference in radius between both lines is represented by $\Delta R$. $\mathrm{R}$ stands for the radius of curvature of the cut. Table 3 offers an overview of the different test values for $\Delta \mathrm{R}$ and $\mathrm{R}$.

Table 3. Tested values for $\mathrm{R}$ and $\Delta \mathrm{R}$

\begin{tabular}{|c|l|}
\hline $\begin{array}{c}\mathrm{R} \\
(\mathrm{mm})\end{array}$ & $\infty, 500,250,100,80,60,50,40$ \\
\hline $\begin{array}{c}\Delta \mathrm{R} \\
(\mathrm{mm})\end{array}$ & $1,2,3,4,5,6,7,8$ \\
\hline
\end{tabular}

The angles of the samples were measured using a Rank Taylor Hobson Talysurf (RTHT) roughness measurement device. This method is indirect since a linear fit is made of the roughness profile and the angle to the horizontal plane is calculated from these data. The angle of the part between line number 5 and number 6 (see Figure 3) is subtracted from the angle of the part between the scan line and the cut, resulting in the bending angle over the scan line.

Experimental results and discussion. Taking the process parameters (see Table 1) and the relatively low thermal diffusivity coefficient of AISI304L $\quad\left(\alpha_{\mathrm{th}}=0.405 \mathrm{e}-5 \mathrm{~m}^{2} / \mathrm{s}\right)$ into account, Temperature Gradient (TG) conditions can be expected, as illustrated in Figure 4: the temperature difference over the sheet at the actual position of the laser beam $(0.015 \mathrm{~m})$ amounts to more than $1000^{\circ} \mathrm{C}$. The figure was obtained using the analytical temperature model of Cheng and Lin [11]. Figures 5a-d present the average values with standard deviation of the tests. Also given are the second-order-fit and the coefficients of determination ( $\mathrm{R}^{2}$-values).

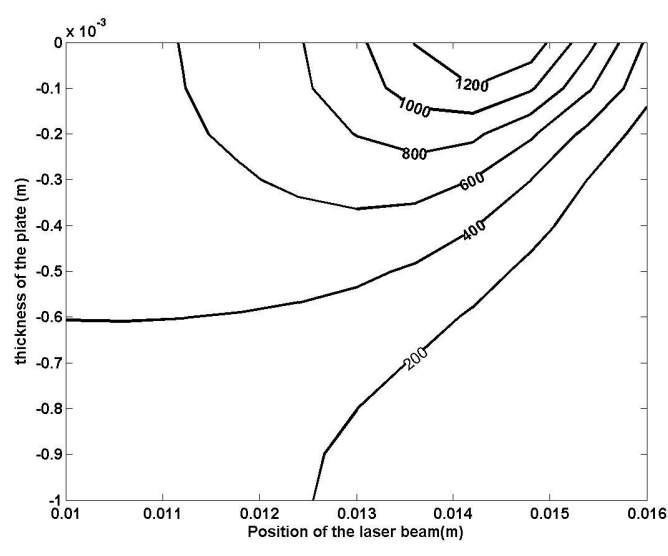

Fig. 4. Temperature course over the thickness of the AISI304L sheet. $\mathrm{P}=6 \mathrm{~kW}$, $\mathrm{DC}=60 \%, \mathrm{v}=5000 \mathrm{~mm} / \mathrm{min}, \mathrm{d}=2.5 \mathrm{~mm}$ and absorption coefficient $=0.10$ 


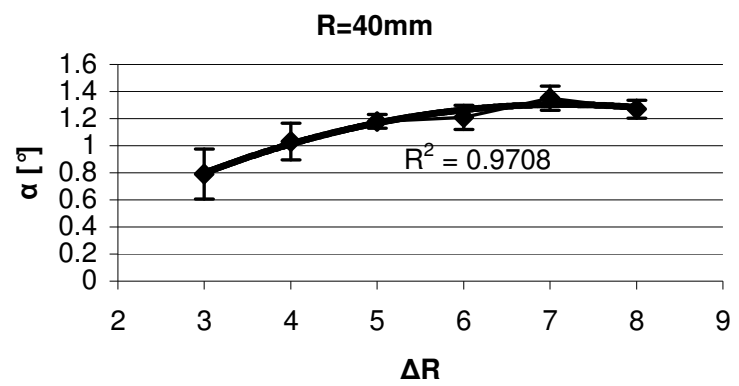

Fig. 5a. Influence of $\Delta \mathrm{R}(\mathrm{mm})$ on bending angle $(\alpha)$ for $R=40$

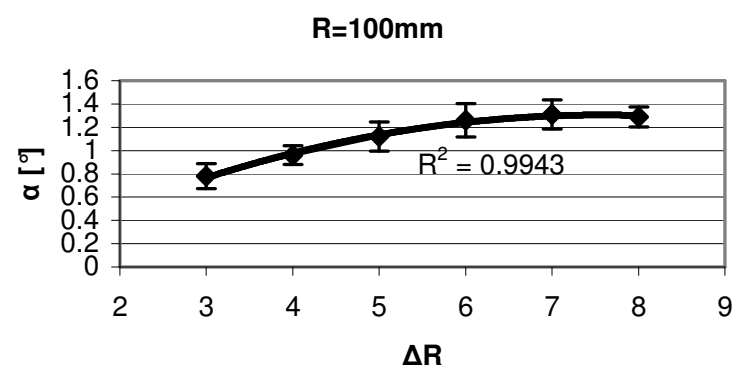

Fig. 5c. Influence of $\Delta \mathrm{R}(\mathrm{mm})$ on bending angle $(\alpha)$ for $R=100$

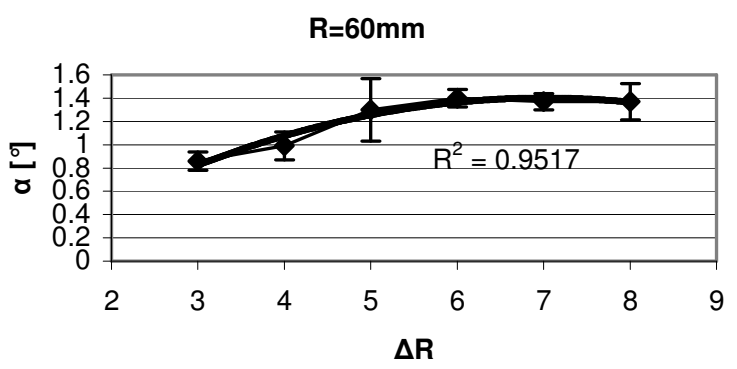

Fig. 5b. Influence of $\Delta \mathrm{R}(\mathrm{mm})$ on bending angle $(\alpha)$ for $R=60$

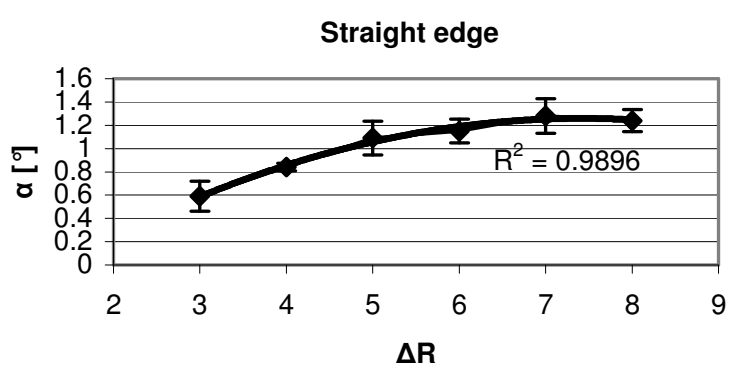

Fig. 5d. Influence of $\Delta \mathrm{R}(\mathrm{mm})$ on bending angle $(\alpha)$ for a straight edge ( $\mathrm{R}$ infinite)

The results for $\mathrm{R}=50,80,250$ and 500 are not presented here, but the trends are similar. For the second-order-fit the coefficients of determination are: $0.9673(\mathrm{R}=50), \quad 0.9275(\mathrm{R}=80)$, $0.9789(\mathrm{R}=250), 0.9266(\mathrm{R}=500)$.

The main findings and interpretations of Figure 5a-d are the following: The results for $\Delta R=1$ and $\Delta R=2$ are not shown. For a laser beam diameter of $2.5 \mathrm{~mm}$, placing the scan line at a distance of 1 $\mathrm{mm}$ from the edge causes a part of the beam to be lost. For $\Delta \mathrm{R}=2$ the beam also proved to be too close to the edge in order to result in a significant angle.

Increasing the distance $\Delta \mathrm{R}$ between the bending line and the edge from 3 to $7 \mathrm{~mm}$, causes the bending angle to systematically grow. For example, the bending angle for $\Delta \mathrm{R}=3$ is smaller than for $\Delta \mathrm{R}=4$ because the temperature gradient over the thickness is smaller for $\Delta \mathrm{R}=3$ than for $\Delta \mathrm{R}=4$. When the scan line is placed close to the edge, the induced heat from very soon reaches the curved edge, resulting in a more even temperature pattern over the thickness of the sheet than in the case when the scan line is placed further away from the cut. This is also the reason why $\Delta \mathrm{R}=2$ does not provide a significant bending angle.

For $\Delta \mathrm{R}=8$ the bending angle decreases slightly. The angles are somewhere between the values for $\Delta \mathrm{R}=6$ and $\Delta \mathrm{R}=7$. Comparing to $\Delta \mathrm{R}=7$, heat removal from the scanned zone is easier, because of the additional material available between the bending line and the curved edge. The maximum bending angle seems to lie around $\Delta R=7$. Possibly for $\Delta R=7$, the space between the scan line and the edge is large enough for the warmth to be dispersed to obtain a big temperature gradient, but small enough to hold the warmth so that during the next scan a bigger angle can be obtained because of the higher starting temperature. Indeed, even though the TG in both successive scans might be equal, the formed angle will be larger for a higher absolute temperature. The latter can be verified when looking at the analytical equation by F. Vollertsen of the bending angle obtained by the two-layer-model [2,p.108]. This equation contains the coefficient of thermal expansion (CTE) 
divided by the product of density and specific heat. For a slightly higher temperature the density and the specific heat do not change, but the CTE increases and even so does the bending angle.

For all radii of curvature, the influence of $\Delta \mathrm{R}$ on the bending angle is dominant to the influence of $\mathrm{R}$. The scatter on the results has the same order of magnitude as the supposed influence of $\mathrm{R}$ on the bending angle and so these tests could not prove a significant influence of the radius of curvature on the bending angle.

\section{Application: Louver}

An application with bending close to the cut is the forming of a ventilation louver. Such form features are normally made by means of dedicated shape tools on punching machines. It is demonstrated here that this is also possible with a 3-axis laser cutting machine.

The setup is shown in Figure 6. The settings are: power $=6 \mathrm{~kW}$, duty cycle $=45 \%$, velocity $=6500 \mathrm{~mm} / \mathrm{min}$, spot diameter $=3 \mathrm{~mm}$. The sample dimensions are $150 * 70 * 0.8 \mathrm{~mm}$. No coating has been added on the samples. The cuts on the sheet are $110 \mathrm{~mm}$ long and the distance between them is $20 \mathrm{~mm}$. The waiting time between the scans was 23 seconds. The rectangular outer contour was cut after the forming took place. Note that the surrounding surface of the louver surface has been devided into 3 separate zones, facilitating the description. The values for the offset between the starting points of the cut and the scan line $(\Delta x)$ and the (perpendicular) offset between the cut and the scan line $(\Delta y)$ are varied and are shown in Table 4.

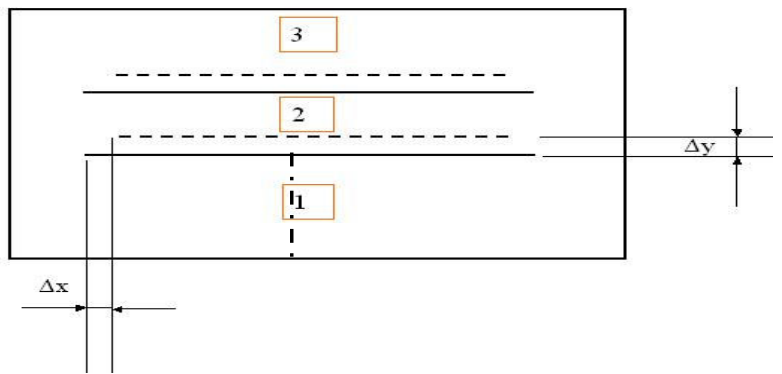

Fig. 6. Scheme for the making of louvers.

Bold: cut; dashed: scanned for forming

Table 4. Tested combinations for $\Delta \mathrm{x}$ and $\Delta \mathrm{y}$ in $\mathrm{mm}$

\begin{tabular}{|l|l|l|l|l|}
\hline$\Delta \mathrm{x}=5, \Delta \mathrm{y}=3$ & $\Delta \mathrm{x}=5, \Delta \mathrm{y}=7$ & $\Delta \mathrm{x}=0, \Delta \mathrm{y}=5$ & $\Delta \mathrm{x}=15, \Delta \mathrm{y}=5$ & $\Delta \mathrm{x}=25, \Delta \mathrm{y}=5$ \\
\hline$\Delta \mathrm{x}=5, \Delta \mathrm{y}=5$ & $\Delta \mathrm{x}=5, \Delta \mathrm{y}=9$ & $\Delta \mathrm{x}=10, \Delta \mathrm{y}=5$ & $\Delta \mathrm{x}=20, \Delta \mathrm{y}=5$ & \\
\hline
\end{tabular}

The angles are measured with the RTHT in zone 2 and zone 3 (see Figure 6) along 2 lines perpendicular to the 2 scan lines. The angles measured in zone 3 are always approximately $5 \%$ lower than those measured in zone 2. Each scan line has been scanned 20 times. The laser switches between scan lines after scanning the same scan line twice.

The louver has two states of equilibrium for zone 1, which can both occur with equal probability: either this zone is buckled upwards, or downwards. It is possible to switch between both states just by manually placing a force on zone 1 . Because on an industrial scale it is not desirable to have 2 equilibrium states and because for a louver, functionally seen, an opening is wanted, it was opted to introduce one more scan line in zone 1, perpendicular to the cut (point-dash line in Figure 6) and scanned twice under the same conditions as specified at the beginning of this paragraph. This scan line causes zone 1 always to bend down (since we are working under TGM conditions) and allows to predict the shape obtained after scanning. 
Figure $7 \mathrm{a}$ shows an overview for $\Delta \mathrm{y}$ kept constant to $5 \mathrm{~mm}$, while $\Delta \mathrm{x}$ varies from 0 to 25 , wheras Figure $7 \mathrm{~b}$ shows the results for $\Delta \mathrm{x}$ kept constant to 5 , while $\Delta \mathrm{y}$ is varying from 3 till $9 \mathrm{~mm}$. Both for the angles measured in zone 2 .

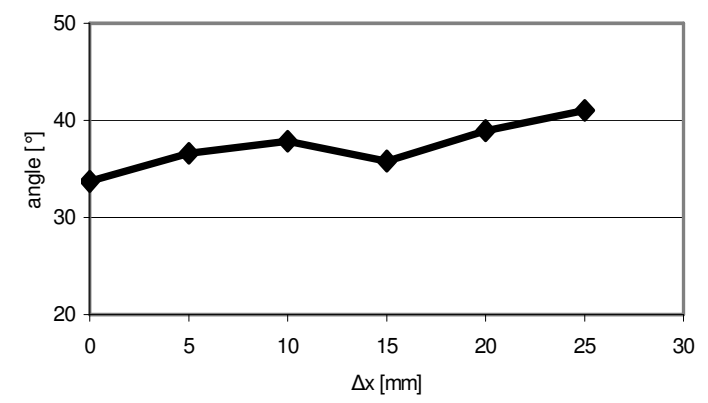

Fig. 7a. Bending angle in zone 2 for $\Delta y=5 \mathrm{~mm}$

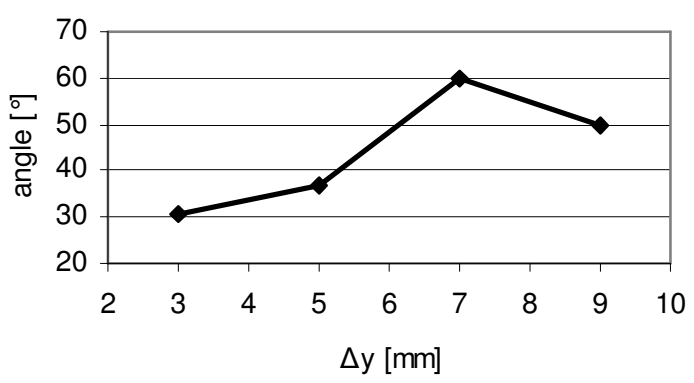

Fig. 7b. Bending angle in zone 2 for $\Delta \mathrm{x}=5 \mathrm{~mm}$

The angle variation for $\Delta \mathrm{y}$ kept constant, where $\Delta \mathrm{x}$ varies from 0 to $25 \mathrm{~mm}$, is less significant than when $\Delta y$ varies with a constant $\Delta x$. The trend in Figure $7 \mathrm{~b}$ is in agreement with the findings of Figure 5 where the bending angle reaches a maximum for $\Delta \mathrm{R}=7$. The trend in Figure $7 \mathrm{a}$ can be summarised as follows: for a large $\Delta \mathrm{x}$ or a shorter bending line, the angle becomes also larger. This could be due to the shorter cooling time between 2 consecutive scans. Although the overall waiting time between the scans is the same, for larger $\Delta x$, the same spot is scanned earlier during the second scan and thus this spot has a higher absolute temperature than for smaller $\Delta \mathrm{x}$, making it easier to obtain deformation. More tests are needed to confirm this conclusion.

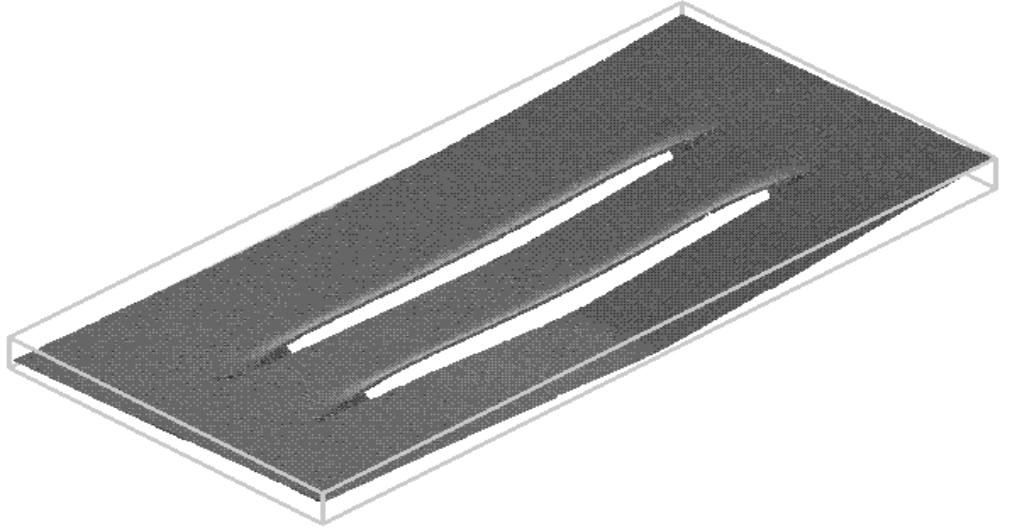

Fig. 8. Rendered mesh of the point cloud, obtained with a line scanning system. The louver shown, is obtained for $\Delta x=\Delta y=5$ $\mathrm{mm}$. The overall unwanted deformation is visualized by a box around the mesh

All the samples show an unwanted deformation, which is more or less spherical. The geometrical information was obtained using a dimensional measurement line scanning system (Metris probe type LC50). A mesh of the obtained point cloud (12700 points) can be seen (Figure 8). The spherical deformation could possibly be removed using another bending mechanism: the upsetting mechanism (UM).

The louver with the smallest overall spherical deformation is considered the most suitable. To draw a conclusion on that, a sphere is fit only through a part of the pointcloud, namely zone 3. This zone can be considered characteristic for the deformation of the complete louver. A sphere with a larger radius indicates a smaller overall deformation for this zone and also for the louver. Table 5 summarises the results of this analysis. 
Table 5. Radii of the spheres for the different settings of $\Delta \mathrm{x}$ and $\Delta \mathrm{y}$

\begin{tabular}{|c|c|c|c|c|c|}
\hline$\Delta \mathrm{x}$ & $\Delta \mathrm{y}$ & $\begin{array}{l}\text { Radius of sphere } \\
{[\mathrm{mm}]}\end{array}$ & $\Delta \mathrm{x}$ & $\Delta \mathrm{y}$ & $\begin{array}{l}\text { Radius of sphere } \\
{[\mathrm{mm}]}\end{array}$ \\
\hline 5 & 3 & 2080 & 10 & 5 & 1019 \\
\hline 5 & 5 & 913 & 15 & 5 & 859 \\
\hline 5 & 7 & 1470 & 20 & 5 & 986 \\
\hline 5 & 9 & 658 & 25 & 5 & 1085 \\
\hline 0 & 5 & 807 & & & \\
\hline
\end{tabular}

It can be concluded that the louver with the smallest $\Delta y$ provides the smallest overall unwanted deformation. This could be expected since the smallest $\Delta y$ also leads to the smallest bending angle (see Figure 7b).

Also for good results, as a rule of thumb, the offset between the scan line and the cut of zone 2 has to be less than one third of the offset between the two cuts: if the bending line would be placed in the middle of the two cuts, quite obviously a symmetrical bending would appear between the two cuts, but unfortunately there would be no opening and so no louver.

Once the forming of 'easy' louvers is under control, we can start thinking of the forming of more complex, customised louvers. As an example the forming of a louver is taken, consisting of 2 arbitrary initials, in this case HV (Figure 9). Only bending lines in one direction are used in order to get a 'controlled' unwanted spherical deformation (see also Figure 8). Furthermore, too long bending lines were avoided, limiting the heat input and deformation. The plate was clamped during forming. The settings are the same as for the other louvers in this paper. In this case $\Delta x=\Delta y=5 \mathrm{~mm}$. Each scan line was scanned 20 times and after every bending line was scanned 5 times, the SOD was corrected automatically.
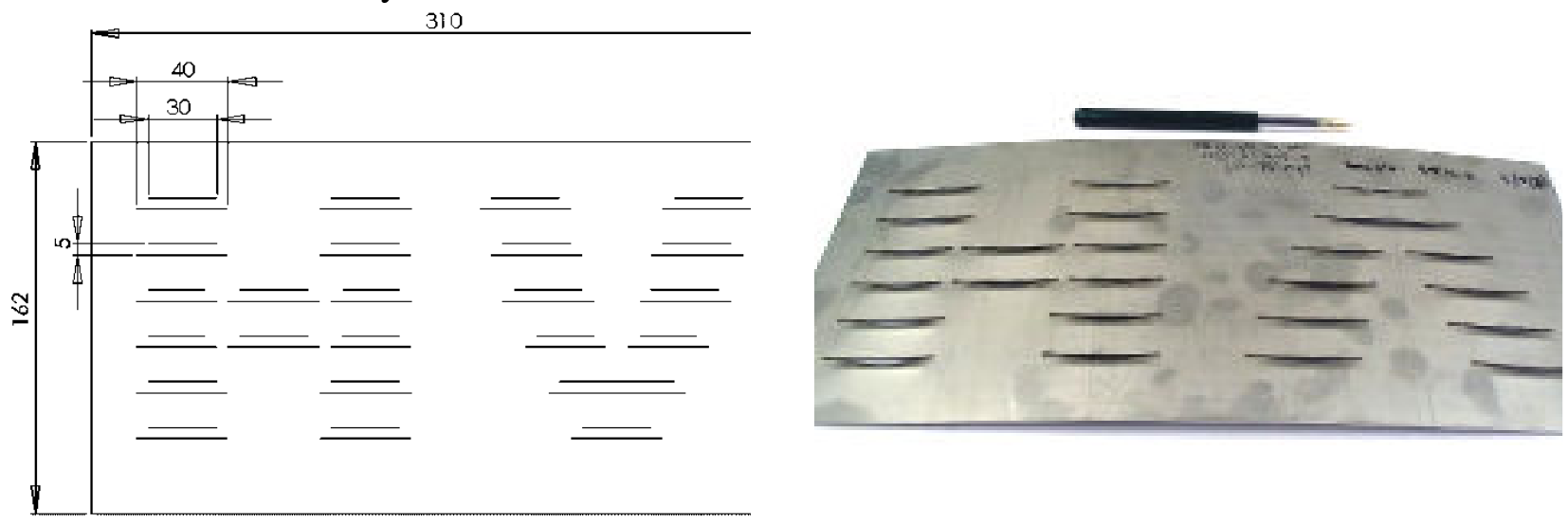

Fig. 9. Customised louver consisting of two arbitrary initials. Left: drawing (in mm). Right: photograph

In order to quantify the unwanted spherical deformation, the same method as above has been used, namely the fitting of a sphere into the pointcloud. The radius of this sphere is $1369 \mathrm{~mm}$ and even though the total number of scan lines is 12 times higher (24), the radius of the sphere stays approximately the same.

\section{Conclusions}

This paper demonstrates that it is possible to generate a 3D feature out of a sheet metal blank using the TGM. As this research was performed on a common 3-axis cutting machine, this widens the application range of this type of machines. 
It was shown that the bending angle grows when the scan line is placed further away from an edge, but reaches a maximum. The radius of curvature has no significant influence on the bending angle.

For the manufacturing of louvers, the following rules of thumb should be respected:

- The offset between the bending line and the cut is best less than $7 \mathrm{~mm}$ in the case where the offset between the cutting is $20 \mathrm{~mm}$.

- The configuration with the scan line closest to the cut gives the smallest overall (unwanted) deformation, but also the smallest (wanted) bending angle.

- For customized louvers, it is better to use shorter bending lines.

\section{References}

[1] M. Geiger and F. Vollertsen: The mechanisms of Laser Forming, Annals of the CIRP Vol. 42/1/1993, pp. 301-304.

[2] F. Vollertsen: Laserstrahlumformen Lasergestützte Formgebung: Verfahren, Mechanismen, Modellierung, 1996, Meisenbach Verlag Bamberg, ISBN 3-87525-071-0.

[3] Y. Namba: Laser forming in space, Int. Conf on lasers '85, ed.: C.P. Wang (1986), pp. 403-407.

[4] J. Lawrence, M.J.J. Schmidt and L. Li: The forming of mild steel plates with a $2.5 \mathrm{~kW}$ high power diode laser, International Journal of Machine Tools \& Manufacture, 2001, Vol 41, pp. 967-977.

[5] J. Bao and Y.L. Lawrence: Analysis and Prediction of edge effects in laser bending, Journal of Manufacturing Science and Engineering, 2001, Vol 123, pp. 53-61.

[6] J. Kim and S.J. Na: Development of irradiation strategies for free curve laser forming, Optics \& Laser Technology, 2003, Vol 35, pp. 605-611.

[7] D. Chen, S. Wu and M. Li: Deformation behaviour of laser curve bending of sheet metals, Journal of Materials Processing Technology, 2004, Vol 148, pp. 30-34.

[8] T. Hennige: Formgebung von Blechen durch Laserstrahlumformen, 2001, Meisenbach Bamberg, ISBN 3-87525-140-7.

[9] J. Magee and T. Hennige: Laser Profile Forming, Sheet Metal, Proceedings of the SheMet International Conference 17-19 april 2000, pp. 301-311.

[10] A. Hutterer and H. Hagenah: Correcting non-circular dents by means of laser beam forming, Proceedings of the LANE 2004, pp. 1071-1081.

[11]P.J. Cheng and S.C. Lin: An Analytical model for the temperature field in the laser forming of sheet metal, Journal of Materials Processing Technology, 2000, Vol 101, pp. 260-267. 
Sheet Metal 2005

doi:10.4028/www.scientific.net/AMR.6-8

Laserforming of 3D Features

doi:10.4028/www.scientific.net/AMR.6-8.425 